International Journal of Applied Mathematics

Volume 33 No. $5 \quad 2020,765-782$

ISSN: 1311-1728 (printed version); ISSN: 1314-8060 (on-line version)

doi: http://dx.doi.org/10.12732/ijam.v33i5.3

\title{
ON A SUBCLASS OF STARLIKE FUNCTIONS ASSOCIATED WITH GENERALIZED CARDIOID
}

\author{
Yuzaimi Yunus $^{1 \S}$, Ajab Bai Akbarally², Suzeini Abdul Halim³ \\ ${ }^{1}$ Faculty of Computer and Mathematical Sciences \\ Universiti Teknologi MARA, Cawangan Melaka \\ Kampus Jasin, 77300 Melaka, MALAYSIA \\ ${ }^{2}$ Universiti Teknologi MARA, 40450 Shah Alam \\ Selangor, MALAYSIA \\ ${ }^{3}$ Institute of Mathematical Sciences \\ Universiti Malaya, 50603 Kuala Lumpur, MALAYSIA
}

\begin{abstract}
The purpose of this paper is to investigate a subclass of analytic functions associated with generalized cardioid in the open unit disk. The geometric properties of functions in the subclass are investigated. Subsequently, the bound for initial coefficients, the Fekete-Szegö inequality and second Hankel determinant inequality for functions belonging to this class are obtained. Furthermore, we find the sharp estimate for Toeplitz determinant, $T_{2}(2)$ for this class.
\end{abstract}

AMS Subject Classification: 30C80, 30C45

Key Words: general cardioid; starlike functions; Hankel determinant; Toeplitz determinant

\section{Introduction}

Let us denote by $\mathcal{A}$ the class of analytic functions $f$ in the open unit disk

Received: March 14, 2020

(C) 2020 Academic Publications

${ }^{\S}$ Correspondence author 
$\mathbb{U}=\{z:|z|<1\}$ of the form

$$
f(z)=z+\sum_{n=2}^{\infty} a_{n} z^{n} .
$$

Denote $\mathcal{S}$ as a subclass of $\mathcal{A}$ consisting of univalent functions. Some subclasses of $\mathcal{S}$ play an important role in geometric function theory such as the class of starlike functions, $\mathcal{S}^{*}$ and class of convex functions, $\mathcal{C}$. These classes are characterized by quantities $z f^{\prime}(z) / f(z)$ and $1+z f^{\prime \prime}(z) / f^{\prime}(z)$ respectively. Many authors have generalized the aforementioned classes namely by applications of operators to obtain new subclasses, for example [28].

A function $f$ is said subordinate to $g$, denoted $f \prec g$, if there is an analytic function $\omega(z)$ defined on $\mathbb{U}$ with $\omega(0)=0$ and $|\omega(z)|<1$ such that $f(z)=$ $g(\omega(z))$ for $z \in \mathbb{U}$. If $g$ is univalent in $\mathbb{U}$ then $f \prec g$ is equivalent to $f(0)=g(0)$ and $f(\mathbb{U}) \subset g(\mathbb{U})$. Ma and Minda [13] gave unified representation of various subclasses of $f$ by using subordination. They introduced the following classes that include some well-known classes:

$$
S^{*}(h)=\left\{f \in A: \frac{z f^{\prime}(z)}{f(z)} \prec h(z)\right\},
$$

and

$$
K(h)=\left\{f \in A: 1+\frac{z f^{\prime \prime}(z)}{f^{\prime}(z)} \prec h(z)\right\} .
$$

The function $h(z)$ maps $\mathbb{U}$ onto a set bounded in the right half-plane. Recently, several authors defined many interesting subclasses of $S^{*}$ by restricting the value of $z f^{\prime}(z) / f(z)$ lying in specific domain in the right half plane. Sokol and Stankiewicz [26] introduced the class $S_{L}^{*}$ consisting of functions $f \in \mathcal{A}$ satisfying $\left|\left(z f^{\prime}(z) / f(z)\right)^{2}-1\right|<1 \quad z \in \mathbb{U}$, where $z f^{\prime}(z) / f(z)$ lies in the domain bounded by the right-half of the lemniscate of Bernoulli. This class can be written in the form of subordination as $S_{L}^{*}:=\mathcal{S}^{*}(\sqrt{1+z})=\left\{f \in \mathcal{A}: z f^{\prime}(z) / f(z) \prec\right.$ $\sqrt{1+z}\}$. Some results associated with this class can be found in $[1,8,17,23]$. Aouf, Dziok and Sokol [3] studied the class $S^{*}\left(q_{c}\right)$, where $q_{c}=\sqrt{1+c z}$ which can be reduced to class $S_{L}^{*}$. Also, Mendiratta, Nagpal and Ravichandran [15] investigated and introduced the class $S_{R L}^{*}$ where $z f^{\prime}(z) / f(z)$ lies in the domain bounded by the left-half shifted lemniscate of Bernoulli. With the same concept, Sharma, Jain and Ravichandran [22] studied the class $S_{C}^{*}$ which lies in the domain bounded by a cardioid. For list of some classes that are defined by subordination, one can see [5, 29].

Motivated by the work of Sharma et al. [22], a class defined by subordination such that $z f^{\prime}(z) / f(z)$ lies in a specific curve in the right half plane is 
introduced. We denote the class as $S_{G C}^{*}(k)$, the class of analytic functions in the unit disk with $z f^{\prime}(z) / f(z)$ lying in the interior of the general cardioid in the right half plane.

Let $h_{k}(z): \mathbb{U} \rightarrow \mathbb{C}$ be a function defined by

$$
h_{k}(z)=1+2 k z+k z^{2}, \quad h(0)=1 \quad \text { where } \quad 0<k \leq 2 / 3 .
$$

We define $S_{G C}^{*}(k)$ as a subclass of $\mathcal{A}$ where

$$
S_{G C}^{*}(k):=S^{*}\left(h_{k}\right)=\left\{f \in \mathcal{A}: \frac{z f^{\prime}(z)}{f(z)} \prec 1+2 k z+k z^{2}\right\} .
$$

A function $f$ belongs to $S_{G C}^{*}(k)$ if and only if $z f^{\prime}(z) / f(z)$ lies in the region bounded by a general cardioid $\Omega_{k}$, on the right half plane given by:

$$
\begin{aligned}
\Omega_{k}:= & \left\{x+i y:\left(x^{2}+y^{2}-2 x+1-5 k^{2}\right)^{2}+\right. \\
& \left.4 k^{2}\left(x^{2}+y^{2}-2 x+1-5 k^{2}\right)-8 k^{3} x-8 k^{4}+8 k^{3}=0\right\},
\end{aligned}
$$

where $0<k \leq 2 / 3$.

The function (2) is univalent in $\mathbb{U}$ and it is easy to see that $h_{k}(\mathbb{U})=\Omega_{k}$, therefore $f(z)$ belongs to $S_{G C}^{*}$ if and only if $z f^{\prime}(z) / f(z) \prec h_{k}$. This gives the structural formula for functions in $S_{G C}^{*}(k)$. A function $f \in S_{G C}^{*}(k)$ if and only if there exists an analytic function $q, q \prec h_{k}$ such that

$$
f(z)=z \exp \left(\int_{0}^{z} \frac{q(t)-1}{t} d t\right) .
$$

Choosing $q=h_{k}$ in (4), we see that the function

$$
f_{0}(z)=z \exp \left(2 k z+\frac{k z^{2}}{2}\right)=z+2 k z^{2}+\left(2 k^{2}+\frac{k}{2}\right) z^{3}+\ldots
$$

belongs to the class $S_{G C}^{*}(k)$.

Interestingly, the main focus of univalent function theory is to investigate on the coefficients of functions. The extensive focus is to estimate the bounds of coefficients and this includes Hankel determinant. Hankel determinant of $f$ was defined by Pommerenke [18] as

$$
H_{q}(n)=\left|\begin{array}{cccc}
a_{n} & a_{n+1} & \cdots & a_{n+q-1} \\
a_{n+1} & a_{n+2} & \cdots & a_{n+q} \\
\vdots & \vdots & \vdots & \vdots \\
a_{n+q-1} & a_{n+q} & \cdots & a_{n+2 q-2}
\end{array}\right|,
$$

where $q \geq 1$ and $n \geq 1$. The uniqueness of Hankel determinant is that it has constant entries along reverse diagonal. The subject of investigation for 
this determinant varied from rate of growth of $H_{q}(n)$ as $n \rightarrow \infty$ ([16]) to determination of precise bounds on $H_{q}(n)$ for some classes for specific $q$ and $n$ in the unit disk, $\mathbb{U}$. For $n=1, q=2$ and $a_{1}=1, H_{2}(1)=\left|a_{3}-a_{2}^{2}\right|$ and the sharp inequality $H_{2}(1)=\left|a_{3}-a_{2}^{2}\right| \leq 1$ holds for functions analytic and univalent in $\mathbb{U}$, [4]. The more general functional $\left|a_{3}-\mu a_{2}^{2}\right|$ is known as FeketeSzegö, studied by $[7,10]$. For $n=q=2, H_{2}(2)=\left|a_{2} a_{4}-a_{3}^{2}\right|$ is known as second Hankel determinant. Janteng, Halim and Darus [9] obtained sharp bound for $H_{2}(2)$ for the class $R$. For more details on the class $R$, one can refer to [14]. For other studies about Hankel determinant of various classes, one can refer to $[11,20,21,27]$.

Closely related to Hankel determinant is Toeplitz determinant. The Toeplitz determinant of $f$ for $q \geq 1$ and $n \geq 1$ is defined as

$$
T_{q}(n)=\left|\begin{array}{cccc}
a_{n} & a_{n+1} & \cdots & a_{n+q-1} \\
a_{n+1} & a_{n} & \cdots & a_{n+q} \\
\vdots & \vdots & \vdots & \vdots \\
a_{n+q-1} & a_{n+q} & \cdots & a_{n}
\end{array}\right|
$$

particularly for $q=n=2, T_{2}(2)=a_{2}^{2}-a_{3}^{2} ; q=2, n=3, T_{2}(3)=a_{3}^{2}-a_{4}^{2}$; $q=3, n=1, T_{3}(1)=1+2 a_{2}^{2}+2 a_{2}^{2} a_{3}-a_{3}^{2}$ and $q=3, n=2, T_{3}(2)=\left(a_{2}-\right.$ $\left.a_{4}\right)\left(a_{2}^{2}-2 a_{3}^{2}+a_{2} a_{4}\right)$. For studies on Toeplitz determinant, see [19, 24].

We shall need the following lemmas. Let $P$ be the class of functions $p$ satisfying $\operatorname{Re} p(z)>0, z \in \mathbb{U}$, and in the form

$$
p(z)=1+\sum_{n=1}^{\infty} c_{n} z^{n} .
$$

Lemma 1. ([4]) Let the function $p \in P$ be given by (5), then $\left|c_{n}\right| \leq 2$ for each $n$.

Lemma 2. ([12]) Let the function $p \in P$ be given by (5). Then for some complex valued $x$ with $|x| \leq 1$ and some complex valued $\xi$ with $|\xi| \leq 1$ we have

$$
\begin{gathered}
2 c_{2}=c_{1}^{2}+x\left(4-c_{1}^{2}\right) \\
4 c_{3}=c_{1}^{3}+2\left(4-c_{1}^{2}\right) c_{1} x-c_{1}\left(4-c_{1}^{2}\right) x^{2}+2\left(4-c_{1}^{2}\right)\left(1-|x|^{2}\right) \xi .
\end{gathered}
$$


Lemma 3. ([13]) Let $p \in P$. If $\nu$ is a real parameter then

$$
\left|c_{2}-\nu c_{1}^{2}\right| \leq \begin{cases}-4 \nu+2, & \text { if } \nu \leq 0 \\ 2, & \text { if } 0 \leq \nu \leq 1 \\ 4 v-2, & \text { if } \nu \geq 1\end{cases}
$$

Lemma 4. ([6]) Let $p \in P$ be of the form (5) and $\lambda \in \mathbb{C}$. Then

$$
\left|c_{n}-\lambda c_{k} c_{n-k}\right| \leq 2 \max \{1,|2 \lambda-1|\},
$$

for $1 \leq k \leq n-1$.

Lemma 5. ([2]) For $0<a<\sqrt{2}$, let $r_{a}$ be given by

$$
r_{a}= \begin{cases}\left(\sqrt{1-a^{2}}-\left(1-a^{2}\right)\right)^{1 / 2}, & \text { if } 0<a \leq 2 \sqrt{2} / 3, \\ \sqrt{2}-a, & \text { if } 2 \sqrt{2} / 3 \leq a<\sqrt{2},\end{cases}
$$

then $\left\{w:|w-a|<r_{a}\right\} \subseteq\left\{w:\left|w^{2}-1\right|<1\right\}$.

The aim of this paper is to investigate the geometric properties of functions in the class $S_{G C}^{*}(k)$. We also seek the upper bound for initial coefficients, Fekete-Szegö functional, second Hankel determinant and also several Toeplitz determinants.

\section{Main results}

Firstly, we shall prove the following lemma.

Lemma 6. For $1-k<a<1+3 k$, let $r_{a}$ be given by

$$
r_{a}= \begin{cases}a-1+k, & \text { if } \quad 1-k<a<1+k, \\ 1+3 k-a, & \text { if } \quad 1+k \leq a<1+3 k,\end{cases}
$$

and $R_{a}$ be given by

$$
R_{a}=\left\{\begin{array}{r}
1+3 k-a, \quad \text { if } 1-k<a \leq 1 / 3(k+3), \\
\sqrt{(1-a)(1-a-2 k)+5 k^{2}+k(k+1-a)^{2} /(a-1)}, \\
\text { if } 1 / 3(k+3) \leq a<1+3 k,
\end{array}\right.
$$

then $\left\{w:|w-a|<r_{a}\right\} \subseteq \Omega \subseteq\left\{w:|w-a|<R_{a}\right\}$. 
Proof. Let $\psi(z)=1+2 k z+k z^{2}$. Then on the boundary of $\psi(\mathbb{U})$ we have $\psi\left(e^{i t}\right)=w$. The parametric equations of $w=u+i v$ are given by

$$
u(t)=1+2 k \cos t+k \cos 2 t, \quad v(t)=2 k \sin t+k \sin 2 t .
$$

Since the curve $w=\psi\left(e^{i t}\right)$ is symmetric with respect to the real axis, so it is sufficient to consider the interval $0 \leq t \leq \pi$. The square of the distance from the point $(a, 0)$ to the points on the curve is given by

$$
\begin{aligned}
z(t) & =(u(t)-a)^{2}+(v(t))^{2} \\
& =(1-a+2 k \cos t+k \cos 2 t)^{2}+(2 k \sin t+k \sin 2 t)^{2} \\
& =(1-a)^{2}+5 k^{2}+\left(4 k(1-a)+4 k^{2}\right) \cos t+2 k(1-a) \cos 2 t, \\
z^{\prime}(t) & =-4 k \sin t(1-a+k+2(1-a) \cos t), \\
z^{\prime \prime}(t) & =-\left(4 k(1-a)+4 k^{2}\right) \cos t-8 k(1-a) \cos 2 t .
\end{aligned}
$$

Solving $z^{\prime}(t)=0$ yields stationary points at $t=0, \pi$ and $\cos t=(1-a+$ $k) / 2(a-1)$. First we obtain the radius for a disk, $r_{a}$, so that the disk is inside $\Omega$. Then

Let $1-k<a<1+k, z^{\prime \prime}(\pi)>0$ shows that $z(t)$ is minimum at $t=\pi$.

$$
\begin{aligned}
r_{a} & =\sqrt{z(\pi)} \\
& =\sqrt{k^{2}-2 k(1-a)+(1-a)^{2}} \\
& =k-1+a .
\end{aligned}
$$

For $1+k \leq a<1+3 k, z^{\prime \prime}(0)>0$ then $z(t)$ is minimum at $t=0$. Therefore

$$
\begin{aligned}
r_{a} & =\sqrt{z(0)} \\
& =\sqrt{9 k^{2}+6 k(1-a)+(1-a)^{2}} \\
& =3 k-1+a .
\end{aligned}
$$

Next we determine the radius for a disk, $R_{a}$, so that $\Omega$ lie inside the disk.

Let $1-k<a \leq 1 / 3(3+k), z^{\prime \prime}(0)<0$ shows that $z(t)$ is maximum at $t=0$,

$$
\begin{aligned}
R_{a} & =\sqrt{z(0)} \\
& =\sqrt{9 k^{2}+6 k(1-a)+(1-a)^{2}} \\
& =3 k-1+a .
\end{aligned}
$$


If $1 / 3(3+k) \leq a<1+3 k$, it shown that $z(t)$ is maximum at $t=t_{0}$ where $\cos t_{0}=(1-a+k) / 2(a-1)$. Then

$$
\begin{aligned}
R_{a} & =\sqrt{z\left(t_{0}\right)} \\
& =\sqrt{(1-a)(1-a-2 k)+5 k^{2}+k(1+k-a)^{2} /(a-1)} .
\end{aligned}
$$

Our first theorem is to determine the bounds for $\left|a_{n}\right|$ in the class $S_{G C}^{*}$. The similar method in [25] is used to determine it.

Theorem 7. If $f(z)=z+\sum_{n=2}^{\infty} a_{n} z^{n} \in S_{G C}^{*}$ then

$$
\sum_{n=2}^{\infty}\left(\left(\frac{n}{3 k+1}\right)^{2}-1\right)\left|a_{n}\right|^{2} \leqslant 1-\frac{1}{(3 k+1)^{2}}
$$

Proof. Suppose $f \in S_{G C}^{*}$. Then $z f^{\prime}(z) / f(z)=1+2 k \omega(z)+k \omega^{2}(z)$, where $\omega$ is an analytic function in $\mathbb{U}$ with $\omega(0)=0$ and $|\omega(z)|<1$ for all $z \in \mathbb{U}$. Since $f(z)\left(1+2 k \omega(z)+k \omega^{2}(z)\right)=z f^{\prime}(z)$ and $z=r e^{i \theta}$, thus

$$
\begin{aligned}
2 \pi \sum_{n=1}^{\infty}\left|a_{n}\right|^{2} r^{2 n} & =\int_{0}^{2 \pi}\left|f\left(r e^{i \theta}\right)\right|^{2} d \theta \\
& =\int_{0}^{2 \pi}\left|\frac{r e^{i \theta} f^{\prime}\left(r e^{i \theta}\right)}{1+2 k \omega\left(r e^{i \theta}\right)+k \omega^{2}\left(r e^{i \theta}\right)}\right|^{2} d \theta \\
& \geq \int_{0}^{2 \pi} \frac{\left|r e^{i \theta} f^{\prime}\left(r e^{i \theta}\right)\right|^{2}}{(1+3 k)^{2}} d \theta \\
& =\frac{1}{(3 k+1)^{2}} \int_{0}^{2 \pi}\left|r e^{i \theta} f^{\prime}\left(r e^{i \theta}\right)\right|^{2} d \theta \\
& =\frac{2 \pi}{(3 k+1)^{2}} \sum_{n=1}^{\infty} n^{2}\left|a_{n}\right|^{2} r^{2 n},
\end{aligned}
$$

where $0<r<1$ and $a_{1}=1$. Then, we get

$$
\sum_{n=1}^{\infty}\left|a_{n}\right|^{2} r^{2 n}\left(\left(\frac{n}{3 k+1}\right)^{2}-1\right) \leq 0 .
$$

By letting $r \rightarrow 1^{-}$, yields the required result.

We have the following corollary. 
Corollary 8. If $f(z)=z+\sum_{n=m}^{\infty} a_{n} z^{n} \in S_{G C}^{*}$, then

$$
\left|a_{n}\right| \leq \sqrt{\frac{3 k(2+3 k)}{n^{2}-1-3 k(2+3 k)}} \text { for } \quad n=m>\sqrt{1+3 k} \quad \text { and } \quad m \in \mathbb{N} \text {. }
$$

The next theorem gives the necessary and sufficient condition for special functions $z+A_{n} z^{n}$ to be in the class $S_{G C}^{*}$.

Theorem 9. A function $f(z)=z+A_{n} z^{n} \quad(n=2,3, \ldots)$ belongs to the class $S_{G C}^{*}$ if and only if

$$
\left|A_{n}\right| \leq \frac{1-n+\sqrt{(n-1)^{2}+4 k(k+n-1)}}{2(n+k-1)} .
$$

Proof. The function in the form $f(z)=z+A_{n} z^{n} \in S^{*}$ if $\left|A_{n}\right| \leq 1 / n$. Note that $S_{G C}^{*} \subset S^{*}$. Suppose that $w=z f^{\prime}(z) / f(z)$. Function $w$ maps $\mathbb{U}$ onto the disk

$$
\left|w-\frac{1-n\left|A_{n}\right|^{2}}{1-\left|A_{n}\right|^{2}}\right|<\frac{(n-1)\left|A_{n}\right|}{1-\left|A_{n}\right|^{2}} .
$$

Since $a:=\left(1-n\left|A_{n}\right|^{2}\right) /\left(1-\left|A_{n}\right|^{2}\right)<1$ then by Lemma 6 for the disk to lie inside $\Omega$ if and only if

$$
\begin{aligned}
\frac{\left(n-1\left|A_{n}\right|\right)}{1-\left|A_{n}\right|^{2}} & \leq \frac{1-n\left|A_{n}\right|^{2}}{1-\left|A_{n}\right|^{2}}-1+k \\
& =\frac{(1-k-n)\left|A_{n}\right|^{2}+k}{1-\left|A_{n}\right|^{2}}
\end{aligned}
$$

which gives $(n+k-1)\left|A_{n}\right|^{2}+(n-1)\left|A_{n}\right|-k \leq 0$. Solving the inequality yields the result.

We state the following initial coefficients and Fekete-Szegö estimate for the class.

Theorem 10. Let $f \in S_{G C}^{*}$ be of form (1), then

$$
\begin{gathered}
\left|a_{2}\right| \leq 2 k, \\
\left|a_{3}\right| \leq \frac{k}{2} \begin{cases}2, & \text { if } \quad 0<k \leq \frac{1}{4}, \\
4 k+1, & \text { if } \quad \frac{1}{4} \leq k \leq \frac{2}{3},\end{cases}
\end{gathered}
$$




$$
\left|a_{4}\right| \leq \frac{k}{3} \begin{cases}4 k^{2}-3 k+2, & \text { if } \quad 0<k \leq \frac{1}{3} \\ k(4 k+3), & \text { if } \quad \frac{1}{3} \leq k \leq \frac{2}{3}\end{cases}
$$

and for any non-zero complex number $\mu$

$$
\left|a_{3}-\mu a_{2}^{2}\right| \leq k \max \left\{1,\left|\frac{1}{2}-2(2 \mu-1) k\right|\right\} .
$$

Proof. If $f \in S_{G C}^{*}$, then from the condition (3)

$$
\frac{z f^{\prime}(z)}{f(z)}=1+2 k \omega(z)+k \omega^{2}(z)
$$

where $\omega(z)$ is analytic function in $\mathbb{U}$ with $\omega(0)=0$ and $|\omega(z)|<1$ for all $z \in \mathbb{U}$. Let

$$
p(z)=\frac{1+\omega(z)}{1-\omega(z)}=1+c_{1} z+c_{2} z^{2}+\ldots \quad(z \in \mathbb{U})
$$

then $p$ is analytic and $p \in P$. Rearrange (8)

$$
\omega(z)=\frac{p(z)-1}{p(z)+1}=\frac{1}{2}\left(c_{1} z+\left(c_{2}-\frac{c_{1}^{2}}{2}\right) z^{2}+\left(c_{3}-c_{1} c_{2}+c_{1}^{3}\right) z^{3}+\ldots\right) .
$$

Now, by expanding the right and left sides of (7), we obtain

$$
\frac{z f^{\prime}(z)}{f(z)}=1+a_{2} z+\left(2 a_{3}-a_{2}^{2}\right) z^{2}+\left(3 a_{4}-3 a_{2} a_{3}+a_{2}^{3}\right) z^{3}+\ldots
$$

and

$$
1+2 k \omega(z)+k \omega^{2}(z)=1+k c_{1} z+k\left(c_{2}-\frac{c_{1}^{2}}{4}\right) z^{2}+k\left(c_{3}-\frac{c_{1} c_{2}}{2}\right) z^{3}+\ldots .
$$

Then, by comparing (9) and (10) we get

$$
\begin{gathered}
a_{2}=k c_{1}, \\
a_{3}=\frac{k}{2}\left(c_{2}-\left(\frac{1}{4}-k\right) c_{1}^{2}\right), \\
a_{4}=\frac{k}{3}\left(c_{3}-\frac{1}{2}(1-3 k) c_{1} c_{2}+\frac{k}{8}(4 k-3) c_{1}^{3}\right),
\end{gathered}
$$

From well known Lemma 1 and (11), we get

$$
\left|a_{2}\right| \leq 2 k \text {. }
$$


From (12), we have

$$
\left|a_{3}\right| \leq \frac{k}{2}\left|c_{2}-\left(\frac{1}{4}-k\right) c_{1}^{2}\right|
$$

Using Lemma 3, we obtain

$$
\left|c_{2}-\left(\frac{1}{4}-k\right) c_{1}^{2}\right| \leq\left\{\begin{array}{lll}
2, & \text { if } & 0<k \leq \frac{1}{4}, \\
4 k+1 & \text { if } & \frac{1}{4} \leq k \leq \frac{2}{3},
\end{array}\right.
$$

with the value $\nu$ from the lemma is $\nu=\left(\frac{1}{4}-k\right)$. Therefore, using (15) and (16) we get

$$
\left|a_{3}\right| \leq \frac{k}{2} \begin{cases}2, & \text { if } \quad 0<k \leq \frac{1}{4}, \\ 4 k+1, & \text { if } \quad \frac{1}{4} \leq k \leq \frac{2}{3} .\end{cases}
$$

Now, we find the estimate bound for $a_{4}$. From (13) and apply triangle inequality, we have

$$
\left|a_{4}\right| \leq \frac{k}{3}\left(\left|c_{3}-\frac{1}{2}(1-3 k) c_{1} c_{2}\right|+\frac{k}{8}(4 k-3)\left|c_{1}\right|^{3}\right) .
$$

Using Lemma 4 yields,

$$
\left|c_{3}-\frac{1}{2}(1-3 k) c_{1} c_{2}\right| \leq \begin{cases}2, & \text { if } \quad 0<k<\frac{1}{3}, \\ 6 k, & \text { if } \quad \frac{1}{3} \leq k \leq \frac{2}{3} .\end{cases}
$$

Then, together from (18) and Lemma 1, we get

$$
\left|a_{4}\right| \leq \frac{k}{3} \begin{cases}4 k^{2}-3 k+2 & \text { if } \quad 0<k \leq \frac{1}{3}, \\ 4 k^{2}+3 k & \text { if } \quad \frac{1}{3} \leq k \leq \frac{2}{3} .\end{cases}
$$

Next, for functional $a_{3}-\mu a_{2}^{2}$ where $\mu$ is non-zero complex number, using (11) and (12), we have

$$
\begin{aligned}
a_{3}-\mu a_{2}^{2} & =\frac{k}{2}\left(c_{2}-\left(\frac{1}{4}-k\right) c_{1}^{2}\right)-\mu k^{2} c_{1}^{2} \\
& =\frac{k}{2}\left(c_{2}-\left(\frac{1}{4}+(2 \mu-1) k\right) c_{1}^{2}\right) .
\end{aligned}
$$

Applying Lemma 4 , with $\lambda=\frac{1}{4}+(2 \mu-1) k$, we obtain

$$
\left|a_{3}-\mu a_{2}^{2}\right| \leq k \max \left\{1,\left|\frac{1}{2}-2(2 \mu-1) k\right|\right\} .
$$


In the next theorem we look at the second Hankel determinant bound for functions in $S_{G C}^{*}$.

Theorem 11. Let $f$ of the form (1) be in $S_{G C}^{*}$. Then

$$
\left|a_{2} a_{4}-a_{3}^{2}\right| \leq k^{2}
$$

Proof. Substitute (11), (12) and (13) into the functional $a_{2} a_{4}-a_{3}^{2}$, we have

$$
\begin{aligned}
a_{2} a_{4}-a_{3}^{2}= & \frac{k^{2}}{3} c_{1}\left(c_{3}-\frac{1}{2}(1-3 k) c_{1} c_{2}+\frac{k}{8}(4 k-3) c_{1}^{3}\right) \\
& \quad-\frac{k^{2}}{4}\left(c_{2}^{2}-2 c_{1}^{2} c_{2}\left(\frac{1}{4}-k\right)+\left(\frac{1}{4}-k\right)^{2} c_{1}^{4}\right) \\
= & \frac{k^{2}}{24}\left(8 c_{1} c_{3}-6 c_{2}^{2}-c_{1}^{2} c_{2}-\frac{1}{8}\left(16 k^{2}+3\right) c_{1}^{4}\right) .
\end{aligned}
$$

Applying Lemma 2 and simplifying it,

$$
\begin{aligned}
a_{2} a_{4}-a_{3}^{2} & =\frac{k^{2}}{24}\left(8 c_{1} c_{3}-6 c_{2}^{2}-c_{1}^{2} c_{2}-\frac{1}{8}\left(16 k^{2}+3\right) c_{1}^{4}\right) \\
& =\frac{k^{2}}{24}\left(8 c _ { 1 } \frac { 1 } { 4 } \left(c_{1}^{3}+2 c_{1}\left(4-c_{1}^{2}\right) x-c_{1}\left(4-c_{1}^{2}\right) x^{2}\right.\right. \\
& \left.+2\left(4-c_{1}^{2}\right)\left(1-|x|^{2}\right) z\right)-\frac{6}{4}\left(c_{1}^{4}+2 c_{1}^{2}\left(4-c_{1}^{2}\right) x+x^{2}\left(4-c_{1}^{2}\right)^{2}\right) \\
& \left.-\frac{c_{1}^{2}}{2}\left(c_{1}^{2}+x\left(4-c_{1}^{2}\right)\right)-\frac{1}{8}\left(16 k^{2}+3\right) c_{1}^{4}\right) \\
& =\frac{k^{2}}{24}\left(2 c_{1}^{4}-\frac{3}{2} c_{1}^{4}-\frac{1}{2} c_{1}^{4}+4 c_{1}^{2}\left(4-c_{1}^{2}\right) x-3 c_{1}^{2}\left(4-c_{1}^{2}\right) x\right. \\
& -\frac{1}{2} c_{1}^{2}\left(4-c_{1}^{2}\right) x-2 c_{1}^{2}\left(4-c_{1}^{2}\right) x^{2}-\frac{3}{2}\left(4-c_{1}^{2}\right)^{2} x^{2} \\
& \left.+4 c_{1}\left(4-c_{1}^{2}\right)\left(1-|x|^{2}\right) z\right) \\
& =\frac{k^{2}}{24}\left(-\frac{1}{8}\left(16 k^{2}+3\right) c_{1}^{4}+\frac{1}{2} c_{1}^{2}\left(4-c_{1}^{2}\right) x\right. \\
& \left.-\frac{1}{2}\left(c_{1}^{2}+12\right)\left(4-c_{1}^{2}\right) x^{2}+4 c_{1}\left(4-c_{1}^{2}\right)\left(1-|x|^{2}\right) z\right)
\end{aligned}
$$

Using Lemma 1 and assuming without loss generality $c=c_{1} \in[0,2]$. Applying 
triangle inequality, we obtain

$$
\begin{aligned}
\left|a_{2} a_{4}-a_{3}^{2}\right| & \leq \frac{k^{2}}{24}\left(\frac{1}{8}\left(16 k^{2}+3\right) c^{4}+\frac{1}{2} c^{2}\left(4-c^{2}\right)|x|\right. \\
& \left.+\frac{1}{2}\left(c^{2}+12\right)\left(4-c^{2}\right)|x|^{2}+4 c\left(4-c^{2}\right)\left(1-|x|^{2}\right)\right):=G(c,|x|) .
\end{aligned}
$$

Differentiate $G(c,|x|)$ with respect to $|x|$, we get

$$
\frac{\partial G(c,|x|)}{\partial|x|}=\frac{1}{2} c^{2}\left(4-c^{2}\right)+\left(4-c^{2}\right)\left(c^{2}-8 c+12\right)|x| .
$$

It is clear that $\frac{\partial G(c,|x|)}{\partial|x|}>0$, which shows that $G(c,|x|)$ is an increasing function with $|x|$ in closed interval $|x| \in[0,1]$. Therefore, $G(c,|x|) \leq G(c, 1)$, and

$$
\begin{aligned}
\max G(c,|x|)= & G(c, 1) \\
= & \frac{k^{2}}{24}\left(\frac{1}{8}\left(16 k^{2}+3\right) c^{4}+16 c-4 c^{3}+\frac{1}{2} c^{2}\left(4-c^{2}\right)\right. \\
& \left.\quad+\frac{1}{2}\left(4-c^{2}\right)\left(c^{2}-8 c+12\right)\right) \\
= & \frac{k^{2}}{24}\left(\frac{1}{8}\left(16 k^{2}-5\right) c^{4}-2 c^{2}+24\right):=F(c), \quad \text { (say) }
\end{aligned}
$$

where $k \in(0,2 / 3]$ is a constant. Then

$$
\begin{aligned}
F^{\prime}(c) & =\frac{k^{2}}{24}\left(\frac{1}{2}\left(16 k^{2}-5\right) c^{3}-4 c\right) \\
& =\frac{k^{2} c}{48}\left(\left(16 k^{2}-5\right) c^{2}-8\right), \\
F^{\prime \prime}(c) & =\frac{k^{2}}{48}\left(3\left(16 k^{2}-5\right) c^{2}-8\right) .
\end{aligned}
$$

The critical numbers are

$$
c=0 \quad \text { and } \quad c=c^{*}=\sqrt{\frac{8}{16 k^{2}-5}} .
$$

For $c^{*}=\sqrt{\frac{8}{16 k^{2}-5}}$,

$$
F^{\prime \prime}\left(c^{*}\right)=\frac{k^{2}}{48}\left(3\left(16 k^{2}-5\right) \frac{8}{16 k^{2}-5}-8\right)
$$




$$
=\frac{k^{2}}{3}>0
$$

then relative minimum occurs at $c=\sqrt{\frac{8}{16 k^{2}-5}}$.

For $c=0$,

$$
\begin{aligned}
F^{\prime \prime}(0) & =\frac{k^{2}}{48}\left(3\left(16 k^{2}-5\right)(0)-8\right) \\
& =\frac{-k^{2}}{6}<0
\end{aligned}
$$

then relative maximum occurs at $c=0$ and the relative maximum is

$$
\begin{aligned}
G(0,1)=F(0) & =\frac{k^{2}}{24}\left(\frac{1}{8}\left(16 k^{2}-5\right)(0)-2(0)+24\right) \\
& =k^{2} .
\end{aligned}
$$

To find the absolute maximum, we also check at the endpoint $c=2$.

$$
\begin{aligned}
G(2,1)=F(2) & =\frac{k^{2}}{24}\left(\frac{1}{8}\left(16 k^{2}-5\right)(16)-2(4)+24\right) \\
& =\frac{4}{3} k^{4}+\frac{k^{2}}{4} .
\end{aligned}
$$

For $k \in(0,2 / 3], \quad k^{2}>\frac{4}{3} k^{4}+\frac{k^{2}}{4}$. Thus, $\left|a_{2} a_{4}-a_{3}^{2}\right| \leq k^{2}$.

The following are Toeplitz determinants bound for functions in $S_{G C}^{*}$. Since the functional in Toeplitz determinants are not rotationally invariant, the assumption $c \in[0,2]$ is invalid.

Theorem 12. Let $f$ be of the form (1) be in the class $S_{G C}^{*}$, then

$$
\left|T_{2}(2)\right|=\left|a_{2}^{2}-a_{3}^{2}\right| \leq \begin{cases}5 k^{2}, & \text { if } 0<k \leq \frac{1}{4} \\ 4 k^{2}+\frac{k^{2}}{4}(4 k+1)^{2}, & \text { if } \frac{1}{4} \leq k \leq \frac{2}{3}\end{cases}
$$

with $0<k \leq 2 / 3$. The inequality is sharp for the function $f(z)=z e^{k\left(2 z+z^{2} / 2\right)}$.

Proof. By using the triangle inequality,(14) and (17), we obtain

$$
\begin{aligned}
\left|T_{2}(2)\right| & =\left|a_{2}^{2}-a_{3}^{2}\right| \leq\left|a_{2}\right|^{2}+\left|a_{3}\right|^{2} \\
& \leq \begin{cases}4 k^{2}+k^{2}, & \text { if } \quad 0<k \leq \frac{1}{4}, \\
4 k^{2}+\frac{k^{2}}{4}(4 k+1)^{2}, & \text { if } \quad \frac{1}{4} \leq k \leq \frac{2}{3}\end{cases}
\end{aligned}
$$


Theorem 13. Let $f$ be of the form (1) be in the class $S_{G C}^{*}$, then

$$
\left|a_{3}^{2}-a_{4}^{2}\right| \leq \begin{cases}k^{2}+\frac{k^{2}}{9}\left(4 k^{2}-3 k+2\right)^{2}, & \text { if } \quad 0<k \leq \frac{1}{4} \\ \frac{k^{2}}{4}(4 k+1)^{2}+\frac{k^{2}}{9}\left(4 k^{2}-3 k+2\right)^{2}, & \text { if } \quad \frac{1}{4} \leq k \leq \frac{1}{3} \\ \frac{k^{2}}{4}(4 k+1)^{2}+\frac{k^{2}}{9}\left(4 k^{2}-3 k\right)^{2}, & \text { if } \frac{1}{3} \leq k \leq \frac{2}{3}\end{cases}
$$

Proof. Using the same concept of proving as in Theorem 12, by applying triangle inequality,(17) and (19), we have

$$
\begin{aligned}
\left|T_{2}(3)\right| & =\left|a_{3}^{2}-a_{4}^{2}\right| \leq\left|a_{3}\right|^{2}+\left|a_{4}\right|^{2} \\
& \leq \begin{cases}k^{2}+\frac{k^{2}}{9}\left(4 k^{2}-3 k+2\right)^{2}, & \text { if } 0<k \leq \frac{1}{4}, \\
\frac{k^{2}}{4}(4 k+1)^{2}+\frac{k^{2}}{9}\left(4 k^{2}-3 k+2\right)^{2}, & \text { if } \quad \frac{1}{4} \leq k \leq \frac{1}{3}, \\
\frac{k^{2}}{4}(4 k+1)^{2}+\frac{k^{2}}{9}\left(4 k^{2}+3 k\right)^{2}, & \text { if } \quad \frac{1}{3} \leq k \leq \frac{2}{3} .\end{cases}
\end{aligned}
$$

Theorem 14. Let $f$ be of the form (1) be in the class $S_{G C}^{*}$, then

$$
\begin{aligned}
& \left|1-2 a_{2}^{2}+2 a_{2}^{2} a_{3}-a_{3}^{2}\right| \\
& \leq \begin{cases}1+8 k^{2}+k^{2} \max \left\{1,\left|1-\frac{k}{2}\left(1+12 k^{2}\right)\right|\right\}, & \text { if } 0<k<\frac{1}{4}, \\
1+8 k^{2}+\frac{k^{2}}{2}(4 k+1) \max \left\{1,\left|1-\frac{k}{2}\left(1+12 k^{2}\right)\right|\right\}, & \text { if } \frac{1}{4} \leq k \leq \frac{2}{3} .\end{cases}
\end{aligned}
$$

Proof. Since $f \in S_{G C}^{*}$ is of the form (1) and applying triangle inequality then

$$
\left|T_{3}(1)\right|=\left|1-2 a_{2}^{2}+2 a_{2}^{2} a_{3}-a_{3}^{2}\right| \leq 1+2\left|a_{2}\right|^{2}+\left|a_{3}\right|\left|a_{3}-2 a_{2}^{2}\right|
$$

Next, applying Fekete-Szegö functional with $\mu=2$ we have

$$
\left|a_{3}-2 a_{2}^{2}\right| \leq k \max \left\{1,\left|1-\frac{k}{2}\left(1+12 k^{2}\right)\right|\right\} \text {. }
$$

By considering (14), (17) and (21), we conclude that the proof is complete.

Theorem 15. For $f \in S_{G C}^{*}$ of the form (1) then

$$
\left|T_{3}(2)\right|=\left|\left(a_{2}-a_{4}\right)\left(a_{2}^{2}-2 a_{3}^{2}+a_{2} a_{4}\right)\right|
$$




$$
\leq\left\{\begin{array}{llc}
6 k^{2}\left(2 k+\frac{k}{3}\left(4 k^{2}-3 k+2\right)\right), & \text { if } & 0<k \leq \frac{1}{4}, \\
\left(2 k+\frac{k}{3}\left(4 k^{2}-3 k+2\right)\right)\left(5 k^{2}+\frac{k^{2}}{4}(4 k+1)^{2}\right), & \text { if } & \frac{1}{4} \leq k \leq \frac{1}{3}, \\
\left(2 k+\frac{k}{3}\left(4 k^{2}+3 k\right)\right)\left(5 k^{2}+\frac{k^{2}}{4}(4 k+1)^{2}\right), & \text { if } & \frac{1}{3} \leq k \leq \frac{2}{3} .
\end{array}\right.
$$

Proof. Let $f \in S_{G C}^{*}$ be of the form (1), then

$$
\begin{aligned}
\left|T_{3}(2)\right| & =\left|\left(a_{2}-a_{4}\right)\left(a_{2}^{2}-2 a_{3}^{2}+a_{2} a_{4}\right)\right| \\
& =\left|a_{2}-a_{4}\right|\left|a_{2}^{2}-2 a_{3}^{2}+a_{2} a_{4}\right| \\
& =\left|a_{2}-a_{4}\right|\left|a_{2}^{2}-a_{3}^{2}-a_{3}^{2}+a_{2} a_{4}\right| \\
& \leq\left|a_{2}-a_{4}\right|\left(\left|a_{2}^{2}-a_{3}^{2}\right|+\left|a_{2} a_{4}-a_{3}^{2}\right|\right) .
\end{aligned}
$$

Clearly, by triangle inequality $\left|a_{2}-a_{4}\right| \leq\left|a_{2}\right|+\left|a_{4}\right|$. Therefore

$$
\begin{aligned}
\left|a_{2}-a_{4}\right| \leq\left|a_{2}\right|+\left|a_{4}\right| & \begin{cases}2 k+\frac{k}{3}\left(4 k^{2}-3 k+2\right), & \text { if } 0<k \leq \frac{1}{3}, \\
2 k+\frac{k}{3}\left(4 k^{2}+3 k\right), & \text { if } \quad \frac{1}{3} \leq k \leq \frac{2}{3}\end{cases}
\end{aligned}
$$

Also, $\left|a_{2}^{2}-a_{3}^{2}-a_{3}^{2}+a_{2} a_{4}\right| \leq\left|a_{2}^{2}-a_{3}^{2}\right|+\left|a_{2} a_{4}-a_{3}^{2}\right|$. Then by (20) and Theorem 12, we obtain

$$
\begin{aligned}
\left|a_{2}^{2}-a_{3}^{2}-a_{3}^{2}+a_{2} a_{4}\right| & \leq\left|a_{2}^{2}-a_{3}^{2}\right|+\left|a_{2} a_{4}-a_{3}^{2}\right| \\
& \leq\left\{\begin{array}{lll}
6 k^{2}, & \text { if } & 0<k \leq \frac{1}{4}, \\
5 k^{2}+\frac{k^{2}}{4}(4 k+1)^{2}, & \text { if } & \frac{1}{4} \leq k \leq \frac{2}{3} .
\end{array}\right.
\end{aligned}
$$

By applying together (22) and (23), we complete the proof.

\section{Acknowledgments}

The first and second authors would like to thank Universiti Teknologi MARA for the support in publishing this paper. 


\section{References}

[1] R.M. Ali, N.K. Jain and V. Ravichandran, On the radius constants for classes of analytic functions, Bull. Malays. Math. Sci.Soc, 36, No 1 (2013), 23-38.

[2] R.M. Ali, N.K. Jain and V. Ravichandran, Radii of starlikeness associated with the lemniscate of Bernoulli and left-half plane, Applied Mathematics and Computation, 218, No 11 (2012), 6557-6565.

[3] M.K. Aouf, J. Dziok and J. Sokol, On a subclass of strongly starlike functions, Applied Mathematics Letters, 24, No 1 (2011), 27-32.

[4] P.L. Duren, Univalent Functions (Grundlehren der mathematischen Wissenschaften) 259), Springer-Verlag, New York-Berlin-Heidelberg-Tokyo (1983).

[5] J. Dziok, R.K. Raina and J. Sokol, On a class of starlike functions related to a shell-like curve connected with Fibonacci numbers, Mathematical and Computer Modelling 57, (2013), 1203-1211.

[6] I. Efraimidis, A generalization of Livingston's coefficient inequalities for functions with positive real part, Journal of Mathematical Analysis and Applications, 435, No 1 (2016), 369-379.

[7] B.A. Frasin, M. Darus, On the Fekete-Szegö problem, International Journal of Mathematics and Mathematical Sciences, 24, No 9 (2000), 577-581.

[8] S.A. Halim, R. Omar, Applications of certain functions associated with lemniscate Bernoulli, J. Indones. Math. Soc, 18, No 2 (2012), 93-99.

[9] A. Janteng, S.A. Halim and M. Darus, Coefficient inequality for a function whose derivative has a positive real part, Journal of Inequalities in Pure and Applied Mathematics, 7, No 2 (2006), 1-5.

[10] S. Kanas, An unified approach to the Fekete-Szegö problem, Applied Mathematics and Computation, 218, No 17 (2012), 8453-8461.

[11] S.K. Lee, V. Ravichandran and S. Supramaniam, Bounds for the second Hankel determinant of certain univalent functions, Journal of Inequalities and Applications, 2013, No 1 (2013), 281. 
[12] R.J. Libera and E.J. Zlotkiewicz, Coefficient bounds for the inverse of a function with derivative in $P$, In: Proc. of the American Mathematical Society, 87, No 2 (1983), 251-257.

[13] W. Ma, D. Minda, A unified treatment of some special classes of univalent functions, In: Proc. of the Conference on Complex Analysis, Int. Press (1994), 157-169.

[14] T.H. Macgregor, Functions whose derivative has a positive real part, Transactions of the American Mathematical Society, 104, No 3 (1962), 532-537.

[15] R. Mendiratta, S. Nagpal and V. Ravichandran, A subclass of starlike functions associated with left-half of the lemniscate of Bernoulli, International Journal of Mathematics 25, No 9 (2014), 1450090.

[16] K.I. Noor, Hankel determinant problem for the class of functions with bounded boundary rotation, Revue Roumaine de Mathématiques Pures et Appliquées, 28, No 8 (1983), 731-739.

[17] E. Paprocki, J. Sokól, The extremal problems in some subclass of strongly starlike function, Zeszyty Nauk. Politech. Rzeszowskiej Mat., 20 (1996), 89-94.

[18] Ch. Pommerenke, On the coefficients and Hankel determinants of univalent functions, Journal of the London Mathematical Society 1 (1966), 111-122.

[19] C. Ramachandran, D. Kavita, Toeplitz determinant for some subclasses of analytic functions, Global Journal of Pure and Applied Mathematics, 13, No 2 (2017), 785-793.

[20] A.K. Sahoo, J.Patel, Hankel determinant for a class of analytic functions related with lemniscate of Bernoulli, International Journal of Analysis and Applications, 6, No 2 (2014), 170-177.

[21] C. Selvaraj, T.R.K. Kumar, Second Hankel determinant for certain classes of analytic function, International Journal of Applied Mathematics, 28, No 1 (2015), 37-50; doi: 10.12732/ijam.v28i1.4.

[22] K. Sharma, N.K. Jain and V. Ravichandran, Starlike functions associated with a cardioid, Afrika Matematika 27,(2016), 923-939.

[23] K. Sharma, V. Ravichandran, Applications of subordination theory to starlike functions, Bull. Iranian Math. Soc., 42, No 3 (2016), 761-777. 
[24] S. Sivasubramanian, M. Govindaraj and G. Murugusundaramoorthy, Toeplitz matrices whose elements are the coefficients of analytic functions belonging to certain conic domains, International Journal of Pure and Applied Mathematics, 109, No 10 (2016), 39-49.

[25] J. Sokól, Coefficient estimates in a class of strongly starlike functions, Kyungpook Mathematical Journal, 49, No 2 (2009).

[26] J.Sokól, J. Stankiewicz, Radius of convexity of some subclasses of strongly starlike functions, Zeszyty Nauk. Politech. Rzeszowskiej Mat., 19 (1996), 101-105.

[27] D.K. Thomas, The second Hankel determinant for starlike functions of order alpha, arXiv Preprint, arXiv:1508.05839 (2015).

[28] Y. Yunus, A.B. Akbarally and S.A. Halim, Properties of certain subclass of starlike functions defined by a generalized operator, International Journal of Applied Mathematics, 31, No 4 (2018), 597-611; doi: 10.12732/ijam.v3li46.

[29] Y. Yunus, S.A. Halim and A.B. Akbarally,Subclass of starlike functions associated with a limacon, AIP Conference Proc. 1974 (2018), \# 030023; doi: $10.1063 / 1.5041667$. 